\title{
Reconstruction of Martensitic Nucleation Process from Morphological Attributes
}

\author{
M.P. Kashchenko, V.V. Letuchev, I.A. Alexina and A.V. Nefedov \\ Physics Chair, Ural State Wood Technology Academy, Sybirskyi Trakt 37, 620032 Ekaterinburg, Russia
}

\begin{abstract}
Within the framework of the concepts of heterogeneous nucleation and wave growth of martensite the refinement of a technique of account of wave normals $\mathbf{N}_{w}$ to expected habits planes are carried out. The technique allows to connect orientations of $\mathbf{N}_{w}$ with definite areas of nucleation of crystals in an elastic field of defects. The specific results are presented for system $\mathrm{Cu}-\mathrm{Zn}$. As separate rectilinear

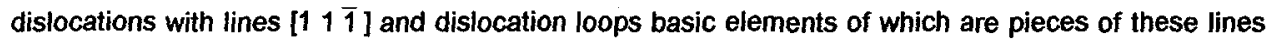
are considered here as probable centers of nucleation. It is shown, that the spectrum of $\mathbf{N}_{\mathbf{w}}$ can be coordinated with experimentally observable space distribution of normals $\mathrm{N}_{e}$ in a vicinity of a pole [2 11 12]. The similar coordination opens additional opportunities to reconstruct a stage of martensite nucleation from morphological attributes and to transform a distribution of habits in the informative parameter.
\end{abstract}

\section{INTRODUCTION}

At the present time, there are serious objections to consider that in many cases the formation of martensitic crystal on a mesoscale is controlled by the atom displacement waves [1]. The excitation of waves occurs at the initial stage through the heterogeneous nucleation of martensite in the elastic fields of some defects. The good results take place already for the individual dislocations $[2,3]$. The initial excited state takes the form of elongated rectangular parallelepiped which is constructed on the eigenvectors $\vec{\xi}_{i}(i=1,2,3)$ of the elastic deformation tensor of dislocation. The respective eigenvalues $\varepsilon_{i}$ satisfy the conditions:

$$
\varepsilon_{1}>0, \quad \varepsilon_{2}<0, \quad\left|\varepsilon_{3}\right|<<\varepsilon_{1}, \varepsilon_{2}
$$

It will be recalled that the fulfillment of (1) ensures an existence of weakly distorted (or invariant if $\varepsilon_{3}=0$ ) planes with normals $\mathbf{N}$. Both $\varepsilon_{i}$ and $\vec{\xi}_{i}$ are the functions of the polar angle $\theta$ which lies in the plane perpendicular to the dislocation line and is measured from the slip plane. Again, $\varepsilon_{j}$ are directly proportional to the ratio $b / r$ where $b$ is the magnitude of Burgers vector of the parent phase dislocation and $r$ is the distance between the dislocation line and the point where the elastic field is calculated. The parameter $b / r \sim a / r$ ( $a$ is the lattice constant) is considered to be small in comparison with unity. It is suggested that there is the excitation of quasilongitudinal

waves vibrating in antiphase and having the normals $\mathbf{n}_{1}, \mathbf{n}_{2}$ being close to $\vec{\xi}_{1}, \vec{\xi}_{2}$ therewith the line of intersection of two wave fronts is parallel to $\vec{\xi}_{3}$. Then the prototype of the habit plane is identified with the plane which is drawn due to the movement of the front intersection line. Such a plane have the normal

$$
\mathbf{N}_{\mathbf{w}} \| \mathbf{n}_{2} \pm x \mathbf{n}_{1}, \quad\left|\mathbf{n}_{1,2}\right|=1, \quad x=c_{2} / c_{1} .
$$

where $c_{1}, c_{2}$ are the velocities of quasilongitudinal waves. Because one of the waves transfers the compression deformation and the second - the tension one [1], the deformation of the kind (1) will be transferred in the wave regime. 
Previously the concrete calculations of $\mathbf{N}$ used the approach:

$$
N \| \vec{\xi}_{2} \pm k \vec{\xi}_{1}, \quad k \approx\left(\varepsilon_{1} /\left|\varepsilon_{2}\right|\right)^{12}
$$

which is formally quite reasonable for $\left|\varepsilon_{i}\right| \ll<$. However in this approach the orientation of $N$ is found to be independent on $r$ and, consequently, any possibility to estimate a space localization of initial state gets lost. As a result the analysis was restricted only to the establishment of the martensite nucleus localization on the polar angle $\theta$.

By this means, for each dislocation it is possible to receive a spectrum of $\mathbf{N}$ and $\mathbf{N}_{\mathbf{w}}$, depending on the interval of corners $\theta$ and procedure of a choice of normals $\boldsymbol{n}_{1}$ and $\boldsymbol{n}_{2}$. The good consent between $\mathbf{N}_{\mathrm{W}}$ and the averaged orientations of normals to the observable habits

$N_{e}$ is resulted already in approximation of simple equality $\mathbf{n}_{1}=\vec{\xi}_{1}, \mathbf{n}_{2}=\vec{\xi}_{2}$. Nevertheless, if to put by the purpose the description of all observable distribution of $N_{e}$, it is desirable to specify a procedure of selection $\mathbf{n}_{1}, \mathbf{n}_{2}$ and to consider the dependence of orientations $\mathbf{N}_{\mathbf{w}}$, $\mathbf{N}$ on the complete set of coordinates of area of localization of initial exited state in the case of more realistic configuration of dislocation nucleation center. Taking into account a good conformity of results of account for separate rectilinear dislocation, it is natural to consider the dislocation loops, basic elements of which are pieces of lines of appropriate dislocations as dislocation nucleation centers (DNC). The similar approach allows to treat the observable distribution of $\mathbf{N}_{\mathbf{e}}$ as the consequence of varying of coordinates of martensite nucleation areas in the elastic fields of defects.

\section{UPDATING A TECHNIQUE OF ACCOUNT}

The problem of the space localization was discussed in [4] in the context of the existence of minimum critical size of the parent phase grain. Clearly [2], that the area of nucleation is defined by the fulfillment of two conditions which are the condition of threshold deformation in an elastic field of DNC and the condition of generation of waves controlling the martensite crystal growth. We shall remind [1], that the fulfillment of the generation condition is only possible for waves with frequencies smaller then Debay's ones by the order of magnitude. Therefore the critical (minimum) cross size $d$ of initial exited state area, capable to result to growth of a crystal (with the thickness d) in a wave mode makes up tens of lattice parameters. On the other hand, by consideration DNC as of power center in a field of which there is the nucleation of martensite crystal, the size $d$ should be though on the order less than distance $r$ up to DNC [2]. It follows that the minimum values of coordinate $r$ of nucleation area which is physically interesting for realization of the wave growth mechanism make up hundreds of lattice parameters. Means, the indirect data about coordinates of nucleation area at $r \geq 10^{2} a$ may be received by investigating the change of orientations of $\mathbf{N}$ and $\mathbf{N}_{\mathbf{W}}$ depending on coordinates of area of nucleation and comparing their orientations with orientations of $\mathrm{N}_{\mathrm{e}}$.

It is interesting to estimate in this connection how strongly does the space dependence of $\mathbf{N}$ and $\mathbf{N}_{\mathbf{w}}$ influence the selection of probable DNC of martensite? For infinite dislocations in the field of applicability of the theory of elasticity such influence should be weak. To calculate that, it is necessary, at first, to write the expression for $k$ in (3) in the general form:

$$
k=\frac{1-\left|\varepsilon_{2}\right|}{1+\left|\varepsilon_{1}\right|} \cdot\left[\frac{\varepsilon_{1}}{\left|\varepsilon_{2}\right|} \cdot \frac{2+\varepsilon_{1}}{2-\left|\varepsilon_{2}\right|}\right]^{1 / 2}
$$

Secondly, it is necessary to take into account, that the eigenvectors of the deformation tensor $\vec{\xi}_{i}$ undergo the rotation, caused as a consequence of the antisymmetric part $\omega_{i j}$ of the distortion tensor $\chi$. That is, in (3) it is necessary to replace $\vec{\xi}_{\mathbf{i}}$ by $\vec{\xi}_{\mathbf{i}}^{\prime}$ where

$$
\vec{\xi}_{i}^{\prime}=Q \vec{\xi}_{i}, \quad\left|\vec{\xi}_{i}^{\prime}\right|=1 \text {. }
$$


In (5) $Q$ is an orthogonal matrix correlated with $\omega_{i j}$. Then, in the framework of the simplest model of the crystal growth control (the wave normals $n_{1}, n_{2}$ coincide with the orientations of the vectors $\vec{\xi}_{1}^{\prime}, \vec{\xi}_{2}^{\prime}$ ) the expression for $\mathbf{N}_{w}^{\prime}$ is:

$$
\mathbf{N}_{w}^{\prime} \| \vec{\xi}_{2}^{\prime} \pm x^{\prime} \vec{\xi}_{1}^{\prime} \text {, }
$$

Where $x^{\prime}$ is the ratio between the wave velocities in the new directions.

It must be stressed that both the components of the tensor $\omega_{i j}$ and the values $\varepsilon_{i}$ are proportional to the ratio $b / r$, where $b$ is the module of Burgers vector. It is obvious that the essential change of the orientations $N$ and $N_{w}$ compared with the orientations (3) at $r \sim 10^{2} \alpha$ can be obtained only with superdislocations with Burgers vectors $b \sim 10 a$, when $\varepsilon_{i}, \omega_{i j} \sim 0.1$. Certainly, this estimation falls outside the limits of applicability of the theory of elasticity and its use is justified only at a qualitative level in order to reveal the tendency of change of $\mathbf{N}$ and $\mathbf{N}_{\mathbf{w}}$.

In order to describe the procedure of selection of normals $\mathbf{n}_{1}, \mathbf{n}_{2}$, behind the approximation $\mathbf{n}_{1}=\vec{\xi}_{1}^{\prime}, \mathbf{n}_{2}=\vec{\xi}_{2}^{\prime}$, it is possible to use a less rigid condition

$$
\vec{\xi}_{1}^{\prime}\left\|n_{1}+e_{1}, \vec{\xi}_{2}^{\prime}\right\| n_{2}+e_{2} \text {, }
$$

where $e_{1}, e_{2}$ are the vectors of polarization of quasilongitudinal waves. The vector $(n+e)$ sets one of the main directions of deformation tensor of a plane wave. Therefore the condition (7) is carried out for a cone of directions of wave normals $n_{i}$ (close $\vec{\xi}_{i}^{\prime}$ ) of quasilongitudinal waves having the main axis corresponding to a tension (or compression) whose orientation coincides with one of the appropriate axis of the elastic field of DNC. The $n_{i}$ satisfying $(7)$ have been obtained with an angular accuracy of 1 minute (at known elastic modules) by the method of consecutive approximations on the basis of the Christoffel equation. It is obvious, that using the $\mathbf{N}_{w}$ from the formula (2), the condition (7) and with $x^{\prime}$ in place of $x$, results in directions of $\mathbf{N}_{w}$ close to the direction of $\mathbf{N}_{W}^{\prime}$ calculated with the help of (6). Thus, condition (7) reflecting roughly the inheriting by wave controlling process of orientations of main axes of tension and compression in the field of nucleation allow a little to expand the range of deviations $N_{w}$ from $N$.

We shall notice, that the comparison with observable orientation of $\mathbf{N}_{e}$ has to be conducted just for the normal $\mathbf{N}_{\mathbf{w}}$. Really, in the non-uniform elastic field of DNC, strictly speaking, in different areas the different local orientations of $\mathbf{N}$ are realized. So the extensive surfaces of interface contact are formed during propagation of wave controlling process. Nevertheless, it is natural to assume that $\mathbf{N}$ and $\mathbf{N}_{\mathbf{w}}$ are close in the nucleation area and to use this condition to specify the criterion of selection. We shall remind the distortion at deformation with one invariant plane, favorable for the interface contact, is described by the diad

$$
\chi=\mathbf{S} \cdot \mathbf{N},
$$

where $\mathbf{N}$ is the normal to the invariant plane, and $\mathbf{S}$ is the vector of shear. The distortion tensor can always be spread out on the sum of three diads. It is obvious, that if the module of the vector $S$ for one of the diads is greater than two other modules of shear vectors in some area of the elastic field of DNC, then $\chi$ takes a form close to (8). Therefore, the condition $\mathbf{S} \approx \mathbf{S}_{\max }$ can be used as one of a selection criteria of $\mathbf{N}$ (and, hence and $\vec{\xi}_{1}^{\prime}, \vec{\xi}_{2}^{\prime}$ ). The condition of local maximum of relative change of volume $\delta$ could be used as the second criterion of nucleation area selection effective for phase transition of the first order in the rigid lattice of initial phase. It is convenient to consider the results of such approach at the beginning for rectilinear infinite dislocations and then to apply it to the dislocation loops. 


\section{RESULTS AND DISCUSSION}

For infinite rectilinear dislocations in the range of values of the parameter $a / r<0,01$, the most interesting from the point of view of transformation physics, it is necessary to expect the weak dependence of orientations of $\mathbf{N}, \mathbf{N}_{w}$ and $\mathbf{N}_{w}^{\prime}$ on $\alpha / \mathbf{r}$. Therefore, for such dislocations it is possible to limit the comparison of $\mathbf{N}_{e}$ and $\mathbf{N}_{w}$, as functions of parameter $\theta$ calculated by the specified technique.

Let us present here the results for an $\mathrm{Cu}-42 \% \mathrm{Zn}$ alloy which exhibits the shape memory effect and undergoes a cooling-induced bcc-fct martensitic transformation. As was discussed in [5], the dislocations with lines [11 $1 \overline{1}$, planes of sliding (011) and Burgers vectors $b_{1}\left\|[11 \overline{1}], \mathbf{b}_{2}\right\|$

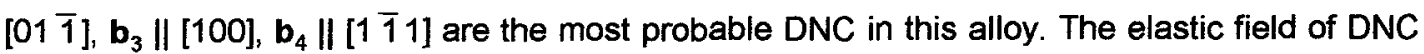
was calculated by the technique [6] for the elastic moduli: $C_{L}=208,15 \mathrm{GPa}, C_{44}=85,50 \mathrm{GPa}$ and $C^{\prime}=6,35 \mathrm{GPa}[7]$. The condition $\mathbf{S}=\mathbf{S}_{\max }[6]$ is carried out, for example, for $\theta=0^{\circ}$, but then $\delta=0$, whereas the nearest local maximum of $\delta$ corresponds to $\theta \approx 13^{\circ}$. The analysis of the dislocation elastic field have shown that the agreement between $N_{e}^{0} \|\left[\begin{array}{lll}2 & 11 & 12\end{array}\right]$ and $N_{w}$ takes place at $\theta \sim 5,4^{\circ}$ for $\delta>0$. Note, that the orientation of the normal of the weakly distorted plane $\mathbf{N}$ nearest to $N_{e}$ is obtained at $\theta=7^{\circ}$. However $\mathbf{N}$ remains orthogonal to the dislocation line and basically cannot coincide with the [2 11 12] direction. Though the distinction between $\mathbf{N}_{w}$ and $\mathbf{N}$ is insignificant, it testifies still for the benefit of selection $\mathbf{N}_{\mathbf{W}}$. Fig. 1 shows the fragment of the stereographic projection for described case. Numbers $i=1,2,3,4$ mark lines, representing the orientations $N_{w}^{\prime}$ when changing $\theta$ in an interval $0^{\circ}-11^{\circ}$ for the infinite dislocations and the intersection determines the pole $N_{e}^{0}$ II [ 211 12]. The narrow areas 1 and 2 in Fig. 1 a gives an idea of the weak dependence on parameter $a / r$, their left borders correspond to $a / r=10^{-4}$ and their right ones to $a / r=10^{-2}$. In Fig. $1 \mathrm{~b}$ the lines 3,4 correspond to $a / r=10^{-2}$. The normals $\mathbf{N}_{w}$ deviate $1,5^{\circ}$ from $\mathbf{N}_{w}^{\prime}$ and are not distinguished in figures. Consequently, the calculated set of $\mathbf{N}_{\mathbf{w}}$ for infinite dislocations is concentrated in a rather narrow area including $\mathbf{N}_{\mathbf{e}}^{0}$ and located, mainly, to the left of the line connecting the poles $\mathbf{N}_{\mathbf{e}}^{0}$ and the [011] direction. The dotted lines in Fig.1a,b determine the orientations of $\mathbf{N}_{w}^{\prime}$ for superdislocations $\left(b_{i} \sim 10 a\right)$ at $r=10^{2} a$. It is clear that for dislocations with vectors $\mathbf{b}_{1}, \mathbf{b}_{2}$ gravitating to screw orientation, the transition to superdislocations (as well as the reduction of $r$ ) reveals the tendency to the displacement of $\mathbf{N}_{w}$ in the area to the right of the line [2 11 12] - [011]. On the contrary, for $\mathbf{b}_{3}, \mathbf{b}_{4}$ gravitating to the edge orientation, the increase of the displacement of $\mathbf{N}_{w}$ to the left from the pole [2 11 12] is observed. It should be noted that the realization of orientations, indicated by a dotted line is obtained most likely from the slow growth of crystals with dislocations lines [11 1 ].

Like the orientation of $\mathbf{N}_{\mathbf{e}}^{0} \|\left[211\right.$ 12] is averaged among the set of $\mathbf{N}_{\mathbf{e}}$, there are orientations located at the left, and to the right of the line $\mathbf{N}_{\mathbf{e}}^{0}-[011]$ (see [8]) and the area of the observable $N_{e}$ to the left of a line $N_{e}^{0}-[011]$ appears wider than the area of orientations of $\mathbf{N}_{w}$ calculated for infinite dislocations. To interpret this result without using the superdislocations, it is necessary to search the explanation from a specification of the DNC configuration. 


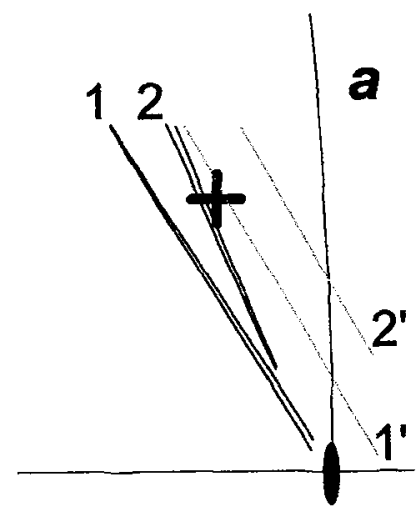

[011]

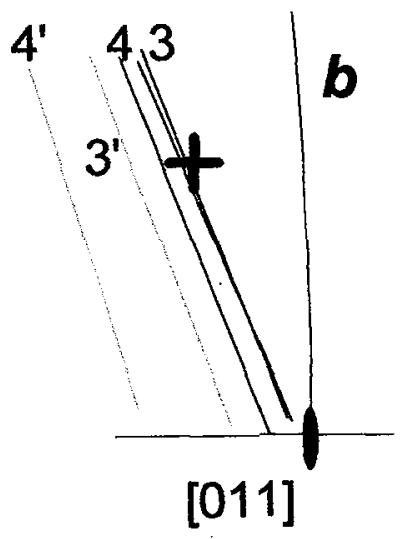

Figure 1: Position of poles $N_{w}^{\prime}$ relative to the pole [2 111 12] for DNC based on infinite dislocations with Burgers vectors: a) $\left.b=b_{1}, b_{2} ; b\right) b=b_{3}, b_{4}$.

As physically acceptable simple configurations, rhomboid and rectangular dislocation loops were considered. The basic elements of loops were pairs of pieces of lines $L_{1}$ along the [11 $\overline{1}$ ] directions. In the case of loops sliding in the plane (011), additional pairs of pieces of lines of

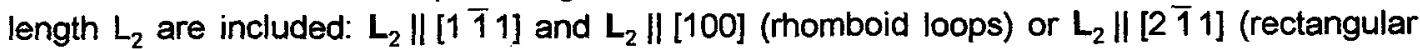
loops). Prismatic loops got out of the rectangular form with $L_{2} \|$ [011]. The origin of the cylindrical coordinate system was located at the center of the loop or in the middle of the line $[11 \overline{1}]$; the polar comer $\theta$ was measured from the loop plane; the axis $\mathrm{OZ}$ was directed along the $[11 \overline{1}]$ direction. For the prismatic loops the cases of surplus ("plate") and missing ("hole") material inside a contour of a loop were considered. The sizes of loops are comparable with the critical size of a grain (about $1 \mu \mathrm{m}$ ). For example, for the results presented below, the lengths of the loop sides were: $L_{1}=7000 a, L_{2}=1000 a$.

Calculations of elastic fields of slipping loops with $r=(200-1000) a$ and small angles $\theta$, lead to a multitude of orientations of $\mathbf{N}_{\mathbf{w}}$ which covers on the stereographic projection the areas 1 and 2 (Fig. 1a). Note that poles to the right of line [011]-[2 1.1 12] are not included.

A different situation corresponds to the prismatic loops. The normals $N_{w}$ for the case $b=b_{4}$, $z=0$ (basis is in the center of the line [11 11$]$ ) are shown in Fig.2: the curves $1(r=200 c)$ and 2 $(r=400 c)$ relate to the "plate"-type loop for angles $\theta$ ranging from $80^{\circ}$ to $90^{\circ}$ and the curves 3 $(r=200 c)$ and $4(r=400 c)$ - to the "hole"-type loop for angles $\theta$ ranging from $90^{\circ}$ to $100^{\circ}$. At $b=b_{3}$ and $r=200 a$ the spectrum of $N_{w}$ is displaced accordingly to the left ("plate") and to the right ("hole") in an appreciable way in comparison with curves 1 and 3 in Fig.2. Thus at $b=b_{3,4}$ for prismatic loops the essential expansion of area of orientations of $\mathbf{N}_{\mathbf{w}}^{\prime}$ in the vicinity of the pole [2 1112 ] is reached in a narrow range of values of $r$ without using superdislocations. In this case the area to the left of the line [011] - [2 11 12] is related with a "plate"-type DNC and to the right - with a "hole"-type DNC.

It should be noted also that for prismatic and sliding loops the orientations of $N_{w}$ in the vicinity of the pole [2 111 12] at $z=0$ found above, are not practically changed for values of $z$ up to $\pm 2500 \mathrm{a}$. This means that the condition for the formation of the initial exited state in the form of elongated parallelepiped are kept in certain areas of the elastic field created by the loop. 


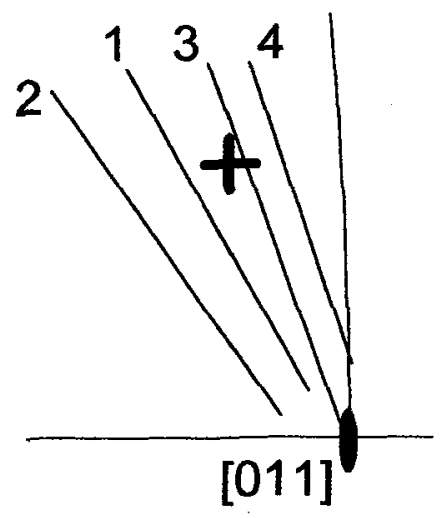

Figure 2: Position of poles $N_{W}^{\prime}$ relative to the pole $\left[\begin{array}{lll}2 & 11 & 12\end{array}\right]$ for DNC based on prismatic "plate"-type (curves 1 and 2) and "hole"-type (curves 3 and 4) dislocation loops with Burgers vectors $b=b_{4}$.

\section{CONCLUSION}

The specification of a technique to account for normals $\mathbf{N}_{\mathbf{w}}$ and the use of dislocation loops as centers for martensite nucleation, expand the opportunities for the interprefation of the observable spectrum of orientations $\mathbf{N}_{e}$. In particular, the results obtained for the $\mathrm{Cu}-\mathrm{Zn}$ system show that martensite crystals with habit plane orientations in the vicinity of the pole [2 $\left[\begin{array}{lll}2 & 11 & 12\end{array}\right]$ can be formed by nucleation in the fast wave mode on distances $r=(200-400) a$ from prismatic dislocation loops (with unit Burgers vectors) whose basic element are lines of the close-packed [11 1 ] direction; in so doing the area to the left of the pole is connected with the nucleation near the "plate"-type loops and the area to the right, with nucleation near the "hole"-type loops. Thus, the comparison of complete sets of experimentally observed orientations of $\mathrm{N}_{\mathrm{e}}$ and calculated wave normals $\mathrm{N}_{\mathrm{w}}$ becomes the tool for reconstruction of a physical picture of the martensite nucleation regime.

\section{Acknowledgments}

The authors would like to thank Prof.A.Planes for useful discussions

\section{References}

[1] Kashchenko M.P., The wave model of martensite growth for $\gamma-\alpha$ transformation in ironbased alloys (UIF Nauka, Ekaterinburg, 1993).

[2] Kashchenko M.P., Letuchev V.V., Konovalov S.V. and Neskoromnyi S.V., Phys. Met. Metallogr., 76(1993) 300-308.

[3] Letuchev V.V., Vereshchagin V.P., Alexina I.V. and Kashchenko M.P., Journal de physique IV, Colloque C8 5(1995) 151-156.

[4] Ivanov Yu.F., Kashchenko M.P., Markov A.B. and Rotshtein V.P., Zhurn. Tekhn. Fiz. 65(1995) No3 98-102.

[5] Kashchenko M.P., Vereshchagin V.P. and Aristova N.V., Phys. Met. Metallogr. 75(1993) 135-138.

[6] Teodosiu C., Elastic models of crystal defects. (Springer, Berlin, 1982).

[7] Murakami Y., Kachi S., Japan. J. Appl. Phys. 13(1974) 1728-1732.

[8] Warlimont H. and Delaey L.,. Martensitic transformation in copper-silver-and gold-based alloys (Pergamon Press Ltd., 1974). 